\title{
Patient Compliance- Practitioner to Patient Communication
}

\author{
Michael F Shaughnessy
}

School of Education, Eastern New Mexico University, USA

Correspondence should be addressed to Michael F Shaughnessy, Michael.Shaughnessy@enmu.edu

Received: March 10, 2021; Accepted: April 3, 2021; Published Date: April 10, 2021

\begin{abstract}
Quite often, health care workers have to prompt, encourage and communicate certain concerns about patient's health and well-being. These concerns can involve medication, diet, exercise, stress, and related factors. This paper will review the issues revolving around the communication and prompting as to life-style changes, dietary changes and medication regimens.
\end{abstract}

\section{KEYWORDS}

Vulnerable individuals; Depression; COVID-19; Technology; App; Digital

\section{SHORT COMMUNICATION}

Patient compliance with medication regimens has been an issue for decades. Over the past 20, 30, 40 years, physicians, nurses, health care workers and others have been attempting to get patients to take their medication, as prescribed, in the proper dosage in the proper way at the proper time, with or without food, and as is often referred to in the field- the right pill in the right way, at the right time.

Clear directions may be on the prescription bottle- but getting patients to follow the directions in a responsible, reliable, dependable fashion has always been challenging. Sometimes patients may already be overwhelmed attempting to cope with their illness. Sometimes they may be intellectually challenged. Often, they misunderstand or are forgetful. In the elderly, there are other cognitive problems impacted memory.

This paper will attempt to provide some suggestions for health care providers. Some may be reiterative. Some Citation: Michael F Shaughnessy, Patient Compliance- Practitioner to Patient Communication. Psychiatry 1(1): 25-28. novel and new. Hills [1] has also explored some concerns about working with the elderly.

1) Eye Contact- When explaining to a patient, the importance of taking a certain medication a certain way, at a certain time, with or without food- the health care provider may need to clearly establish eye contact. Now, that being said, some cultural groups may take offense at this- However, on the other hand, it is critical, and crucial that certain medications be taken consistently. And in some instances, it is imperative that the entire course of medication be taken.

2) Asking for eye contact - "Please- can I have your complete, full, undivided attention for a few minutes. Look me here in the eyes" Health care professionals need to invest in the short-run in order to help the patient in the long run. Maintaining eye contact may indicate the seriousness of the situation or the need for compliance with the medication or diet regimen.

3) Tone of voice - In general, health care professionals are cordial and congenial. However, there are times, when 
a patient needs to know the seriousness of their condition in no uncertain terms. This can be communicated by simply raising one's voice slightly or clearing one's voice before beginning or changing one's body posture----sitting down for example at eye level to discuss the need for patient compliance. Often this construct needs to be clearly explained to certain individuals. For example- "the entire course of this medication MUST, I repeat MUST, dare I say it againMUST be taken in order for it to be effective."

4) Checking understanding - Often the health care provider must actually check that the patient has heard and understands the specific directions regarding the administration of the medication. It is important to ask the patient to repeat what has been said to them and to also on occasion write down on a piece of paper (some individuals react better to a colored piece of paper) the directions.

5) Directives - Directives can be subtle or not so subtle. "This is a fairly inexpensive medication, and if taken as prescribed can be wonderfully effective. If NOT taken consistently, there are other much more expensive alternatives, some of which may, I repeat MAY have side effects...... and be more expensive."

6) Understanding - Often a kind patient, understanding tone of voice can help communicate the difficulty a patient may be having, for example decreasing their salt or sugar intake. Comments, such as "I am aware of the stress that many people face in their lives, and that food is their only source of solace and comfort and consolation." However, I can show you pictures of individuals who have a similar condition and what over-eating has done to their system and veins, arteries, capillaries, aorta etc.

7) Examples - The importance of taking a certain medication cannot be over-estimated. For example, for patients with active epilepsy, missing one dose of their medication can result in a seizure- which could result in a car accident, which could result in hospitalization, which could result in a large hospital bill that they would have to pay off for the rest of their lives. Health care professionals can also indicate in a truthful manner- a prior case that they had wherein a patient did not comply with the medication regimen, and as a result had to undergo hyperbaric oxygen treatment. Now, while this may sound terrible- it is simply providing a certain degree of oxygen in a chamber for a certain type of wound- but often it involves a very long drive to the closest hospital that offers that type of treatment for that specific type of wound.

8) Pictures - Some health care providers offices have pictures of lungs that have been impacted by smoking, or overuse of those 8 hour energy drinks.

9) “La familia es la cosa mas importante” - In certain cultures- the family is the most important thing. Soencouraging patients to take their medication so that they will be able to take care of their family or parents "down the road"is important.

10) Verbiage - Often health care providers need to utilize a good dose of ostentatious rhetoric- to get the message across- for example- My counsel, my guidance, my suggestion- my advice- is to take the medication exactly as directed- and avoid these following fried food or (whatever the patient needs to avoid).

11)Staggering phraseology - I - (wait 5 seconds) want you (wait 10 seconds) to listen (wait 10 seconds) very carefully------(pause for dramatic effect) to what I have to say about your condition. It is imperative that you and practioners can fill in the blanks as appropriate.

12)Physical contact - Some health care providers can reach over and touch the hand of an elderly individual in order to gain their attention and establish eye contact. Touch sends a message of caring- and establishes a warm human contact, that is often lacking in this tele-medicine stuff.

13)Heart vs Head - Often health care providers need to think about what reaches the patient- do they respond to cognition, and thinking and logic and reason and 
rationality or do they respond to feelings and emotions and the affective realm.

14) The more you know - Often the more information a health care provider can provide about what a medication does, the more cooperation that the patient can provide in terms of compliance. Indicating exactly what a certain drug or medication does to the kidney or liver or heart can be important- and also what things (alcohol, tobacco) can counteract the effects of certain drugs.

15)Pharmacists - Often the local pharmacist, if he or she is well known to the patient can provide more intense explanation as to why compliance is so important. A local pharmacist may be a male or female, or could be of a similar or even the same race or ethnicity or cultural background.

16)Consultation - Any health care provider can consult with any individual more knowledgeable about any condition. This information can be relayed in order to indicate to the patient the importance of their following the directives of the physician or other health care professional. Comments such as "I have consulted with a number of colleagues about your condition and they all seem to feel it important that this particular regimen of medication be followed as exactly, and as precisely and completely as indicated. We are trying to increase/ decrease-and then to maintain some homeostasis for your condition.

17)Setting aside time for medication compliance issues The physical presence of a nurse, physician's assistant or oncologist is often a treatment variable. Following a routine examination and documentation, a health care professional can leave the room- and return, specifically to discuss compliance issues. "I am setting aside this time- because it is important that this cream, salve, patch, be administered exactly as directed" is a sample phrase.

18)Terminology - Not all patients may grasp sublingual and may need to be told that the medication is to be placed under the tongue and dissolved.
19)Lecturing is not always a viable approach - Listening to the patient first may prove more beneficial and result in some catharsis that may provide some insight. Some people eat comfort food because they are under unimaginable stress. Some are in unendurable psychological pain. When do you eat, where do you eat, why do you eat are questions that would provide a roadmap for those struggling with obesity and diabetes. Further it could provide a baseline for future intervention. Indeed, some health care workers on first encounter with a patient may need to see that first intake as a major step in rapport building for future endeavors.

20)For some elderly, there are plastic boxes that can be purchased with A.M. and P.M. and the days of the week on them. It may be worthwhile for a nurse or caregiver to employ these plastic boxes to ensure patient compliance with their medication.

21)Cell phone use - In some instances, the elderly can be trained to use the cell phone to call the pharmacy- and provide the prescription number and later in the day pick up their medication, rather than driving to the pharmacy, and becoming exasperated and frustrated waiting for their prescription to be re-filled.

22)Keywords - In terms of working with certain patients, it is important to emphasize key words. The practitioner or health care professional may need to focus on the relationship of one word to another. For example, phenobarbital is a medication used to prevent epilepsy. It must be taken in order to prevent a seizure. And it must be taken consistently. And if you miss a dosage, here is what I want you to do: Here, depending on importance, one can emphasize the needed steps. In other instances, there may be words that need to be explained. For example, there are anti-coagulant drugs that may need to be titrated. Explaining to a client/patient that blood clots can be dangerous and that one needs to have a medication that prevents these blood clots and also this medication may prevent blood clots from reaching the brain. Here linking the 
medication with it's purpose and reason is important and needs to be communicated to the patient.

\section{DIET, STRESS AND SLEEP}

Often health care workers (this included physicians, nurses, dieticians, physicians assistants, OTs, PT's, even pharmacists) feel the need to discuss proper diet, coping with stress and life style changes, sleep and the cessation of negative habits such as smoking, alcohol abuse, and the consumption of sugar, salt, and other items which can negatively impact the functioning of the human body, and offset any medication administered (for example, high blood pressure medication works, but can be offset by bags of potato chips, pretzels, popcorn, and of course French fries which are loaded with salt. Patients have to be educated as to the fact that many things that they consume as loaded with sugar, salt, and other toxic types of things (such as MSG). How to communicate with obese patients, diabetic patients, and pre-diabetic patients is crucial. They may of course, fall back on their youth. They may believe that they are going to live forever. Often the health care worker has to "read the mind" of the 30-yearold diabetic who may not be aware that 31-year-old individuals can go into a diabetic coma and die. Many of the current generation have not encountered death, the Intensive Care Unit or the Emergency Room or many have not even been in a hospital or nursing home. They are oblivious. This cannot be emphasized too much.
Simple things, such as talcum powder, and Weedkillers CAN result in major cancer issues.

\section{Keeping it simple regarding Diet}

Often a person is overweight due to ONE simple food that could be eliminated from their diet. A dietician may be able to find out what that food is that is causing weight gain- or weight maintenance and preventing weight loss.

Often a person is overweight due to the fact that they eat at ONE specific restaurant or fast-food joint on an ongoing basis.

Often a person is overweight in that they quench their thirst using ONE particular soft drink or beverage.

Getting a commitment from a patient to eliminate ONE food, one soft drink may be a major victory. Getting a smoker from 4 packs a day down to ONE pack a day is another success story.

But there has to be commitment.

Wills [2] has cogently explored this realm also. There are often many issues involved in weight loss, diet, exercise and health related concerns.

\section{SUMMARY AND CONCLUSION}

This paper has attempted to review some of the communication issues that can assist health care personnel in acquiring patient compliance with medication regimens.

\section{REFERENCES}

1. Hills L (2012) Working effectively with older patients: 25 do's and don'ts for medical practice employees. Journal of Medical Practice Management 27(4): 222-226.

2. Wills T (2015) Factors relating to motivation to change behaviour in individuals who are overweight: application of the health belief model. Cork Open Research Archive, DN Thesis, University College Cork, Ireland. 